\title{
ON A CASE
}

OF

\section{OBSTRUCTION OF ONE URETER BY A CALCULUS,}

ACCOMPANIED BY COMPLETE SUPPRESSION OF URINE.

BY

RICKMAN J. GODLEE, M.S., F.R.C.S., SURGRON TO UNIVERSITY COLLEGR HOSPITAT.

Received February 6th, 1886-Read March 22nd, 1887.

IT is hoped that this isolated case will be deemed worthy of consideration by the Fellows of the Society on account of the occurrence of certain unusual symptoms which have an important bearing on the diagnosis of this often obscure disease ; but, if this excuse were insufficient, it might be added that the patient was an indefatigable member of our own profession and most anxious that his own sufferings should, if possible, be utilized for the general good.

The only serious illness from which he had suffered occurred during his student days in 1872, when he was about nineteen years of age. He had then a poisoned wound which resulted in symptoms of general septicæmia and was accompanied by left-sided pleurisy and no doubt pericarditis. He was much reduced by this but gradually recov- 
ered, and from that time until June, 1884, he enjoyed very good health interrupted only by occasional attacks of colic, thought by him to be intestinal, and passed over as matters of small consequence. The first of these occurred about 1881; it only lasted for two hours and was relieved by the administration of chloroform. A second, a year later, lasted four hours and yielded to the hypodermic injection of morphia. They both consisted of severe abdominal pain, not localized, and accompanied by much sweating, and on the second occasion by vomiting, but there was nothing in either to suggest the ureter as the seat of the disorder, nor in the appearance of the urine to prompt the patient to examine it. At times, after this second attack, he experienced sharp shooting pains in the right testicle, which he thoughtlessly attributed to a large leftsided varicocele from which he suffered, but otherwise he considered himself quite well.

The illness for which he asked my advice began therefore without any other warning recognised as such on June 4th, 1884, with increased frequency in micturition and a pain in the region of the bladder if he attempted to retain his water. Before this time, and indeed afterwards, he was in the habit of emptying his bladder at rather remarkably long intervals. The trouble in passing water lasted for a week, when on June 11th there was a good deal of blood in the urine, and under the microscope oxalate of lime and perhaps some uric acid crystals were discovered; but next day the urine was normal in the morning though blood again appeared in the evening. On the following day, June 13th, abdominal pain like that from which he had suffered in the two previously described attacks, came on and was accompanied by sickness; it was so severe that he kept his bed on this and the two following days, but on the morning of the 16th it had disappeared and he was getting about again. In the evening of this day, however, it returned with great severity, "doubling him up," and becoming localized in the region of the right kidney. His temperature was now $102^{\circ} \mathrm{F}$. 
I saw him on the evening of the 17th. He was then in great pain of a spasmodic character. There was considerable tenderness over the right kidney, but no tumour was discovered on careful palpation. There were anorexia and nausea, and a temperature of $102^{\circ}$. The urine was clear, but contained a few pus-cells and rather more than a trace of albumen; no casts were seen.

After a few days the pain, which had been somewhat relieved by hot fomentations and morphia, disappeared, and at the same time the temperature, which had remained high, fell to normal and there was a considerable increase of pus in the urine.

As it was assumed that some obstruction to the ureter had been overcome he was allowed to get up and to go out, and it was intended that he should go into the country in order to recruit, but after he had been walking rather more than usual and, it should be added, after the bladder had been sounded without the discovery of a calculus, the pain returned again and the temperature assumed a hectic character, varying from $99^{\circ}$ in the morning to $102^{\circ}$ at night, whilst the urine became less turbid. The pain increased very much and the tenderness reappeared, the former shooting down into the groin, the latter being most marked in the back between the ribs and the right iliac crest. The urine was now noticed to contain flocculent masses, which under the microscope were seen to consist chiefly of long felted chains of short bacteria. It was acid and free from putrefactive smell. It is most probable that it also contained granular and hyaline casts, as they were present in large quantities a few days later, but they were not carefully looked for at this time. The most peculiar feature about the urine-and it is one to which I would direct special attention-was the enormous increase in the amount of albumen without any increase in the quantity of pus, so that by July 12th it had reached the proportion of one half, at which period the granular and hyaline casts were exceedingly abundant.

It seemed almost certain that there was some deeply- 
seated matter in the neighbourhood of the kidney, and therefore on July 13th, after consultation with Dr. Broadbent and Dr. Poore, I made a free incision in the lumbar region, intending to expose the kidney. A deep abscess was found, containing about an ounce of thick pus, situated rather in front of and below the kidney. The tissues were quite confused from old inflammatory changes, and I did not succeed in making out accurately the locality of the kidney itself, though several punctures were made with a needle into what was supposed to be the organ, with the object of discovering the stone which it was thought was present. Looked at in the light thrown upon the case by the post-mortem examination, it is probable that the kidney could not be felt because it was even then a mere soft bag of pus embedded in dense inflammatory tissue, while the stone could not have been struck because it was already half way down the ureter. Had I used a perforated, instead of a solid needle, it is probable that I should have tapped the kidney and by opening it at this time have discovered the whereabouts of the stone. But this was not done, so the search was relinquished, and the anterior part of the wound was sewn up after inserting two large drainage-tubes to the bottom of it.

The amount of albumen after the operation was not constant. On the following morning it was only $\frac{1}{12}$, but it afterwards rose to $\frac{1}{4}$ and $\frac{1}{3}$. It gradually, however, sank to a much smaller quantity, while that of the pus remained the same; and though there was an occasional and unaccountable increase, it may be said that after three days it did not amount to more than the pus would account for.

The casts, which were in enormous abundance on the 14th, had quite disappeared by the 17th.

There was no hæmaturia after the operation, and no urine ever came through the wound. The amount of urine passed in the twenty-four hours now varied from 70 to 110 ounces, the sp. gr. being usually about 1012 to 1014 . 
This part of the case may now be summarised by saying that all the symptoms were at once relieved; the nausea disappeared and the appetite returned; the local pain went away, but some remained for a short time in the groin, preventing the patient from extending his hip, the temperature sank at once to normal, and only once or twice rose above $99^{\circ} \mathrm{F}$. after this, these slight rises being, curiously enough, sometimes associated with a very temporary increase in the amount of albumen in the urine; this, however, gradually fell to about $\frac{1}{20}$, and the pus steadily diminished in amount, but the bacteria were always present, notwithstanding the administration of quinine, benzoic acid, and other drugs supposed to be bactericidal.

He left his bed about August 10th and went into the country about August 16th, at this time complaining only of an occasional slight pain at the tip of the penis after micturition, but principally when the urine happened to be particularly thick.

From this time forward the patient much improved and became as well as he had ever been in recent years, and in fact his weight was more than it had been for a long time. But though between 18th August, 1884, and December, 1885 , he was able to return to his practice, and kept in fair general health, he was always thin and pale, and had much of the aspect of a patient suffering from tubercular disease, though repeated examination failed to discover any evidence of the presence of this condition. He had occasional attacks of colic, always situated on the right side, accompanied generally with some rise of temperature and always with sickness and a temporary clearance of the urine, followed by a discharge of pus when the symptoms abated. These attacks were often preceded by an inability to hold his water, or rather by a frequent and irresistible though painless necessity to micturate, lasting for some days before the onset of acute pain. But for long periods he was quite free from any renal symptoms, except that the urine, though scanty, always contained pus and masses of the minute organisms before referred to, vOL. LXX. 
and a small amount of albumen, perhaps no more than would be accounted for by the pus.

The recurrence of these attacks, while the general health did not suffer, determined me, at his request, to undertake a further operation with the object of removing the stone which I presumed was present in the kidney; but, after his mind was made up, there followed a period of such excellent health that the investigation was put off again and again, and on Thursday, December 10th, 1885, after some of the usual preliminary troubles, the last and fatal attack set in. I have no authentic record of the earlier symptoms, but it seems to have been quite similar to the previous ones and continued until Monday the 14th. During this time he administered to himself hypodermic injections of morphia pretty freely, taking (I think he said) two and a half grains subcutaneously. On the 14th the colic passed away and on the 15th complete suppression of urine set in.

I saw him on the 17 th, when he seemed unaware of the serious import of this new symptom. He had then all the appearance of a patient under the influence of opium. His pupils were small and he was very drowsy, dropping off to sleep whenever he was left undisturbed. His skin was dry and the temperature subnormal ; the pulse hard and incompressible, but the heart-sounds indicating an incomplete systole, a slight systolic mitral murmur which had always been present from the first being audible. The respirations were very slow, the expiration especially being prolonged. Nothing could be felt of either kidney as he lay upon his back, nor was there any local pain or tenderness.

Dr. Broadbent and Mr. Beck saw him with me in the evening, and the question of attempting any further operation was carefully discussed and, as the event showed, unfortunately discarded. We concluded that in all probability the right had for a long time been his only working kidney (the left, it was assumed, having been rendered useless either as the result of calculous or strumous disease), and 
that the present complete suppression resulted from the fact that now the right ureter was at last altogether plugged. We discarded as unlikely the hypothesis of the simultaneous plugging of the two ureters with symptoms referred only to the right side, and also the possibility of the suppression being merely functional and sympathetic with the acute trouble on the right side; but I have no doubt that we should have paid more attention to the fact that during the previous attack a fair quantity of moderately healthy urine had been passed whilst there was every reason to suppose that the right ureter was completely or almost completely plugged. It was therefore decided that if anything were to be done it should consist in cutting down on the right kidney, but that the risk of administering chloroform in what appeared to be an advanced state of uræmia (with convulsive symptoms probably imminent) was too great to allow of an operation which, as we thought, could at best only prolong life for a short time. At this time I confess that I came over to the diagnosis of strumous kidney, and was thus quite willing to fall in with the general conclusion. We contented ourselves, therefore, with giving hot-air baths, which produced a copious perspiration, with opening the bowels, which were remarkably sluggish, as indeed were all the functions of the body, and with administering copious warm water enemata in the hope of stimulating the action of the kidneys in case the suppression should after all be functional. For nearly a week the suppression was complete, viz. from the Tuesday till the following Monday morning. During this time the uræmic symptoms became more and more pronounced and we were expecting momentarily, the final convulsion. But on Saturday the 19 th, as he lay on his right side, I was able distinctly to feel the left kidney, which seemed to be slightly tender. I therefore punctured it with an aspirator needle through a small incision in the loin, and, as the autopsy showed, did actually pierce the lowest calyx of the pelvis, either on this occasion or on the following morning, when the process was repeated, but no water was drawn 
off. In the early morning of Monday the 21 st he passed $14 \mathrm{oz}$. of urine, thick and turbid with much tenacious pus, overloaded with albumen (four fifths), but with a very small amount of urea $(0.8$ per cent.). 'This, no doubt, came from the right kidney as will be afterwards seen. In the evening of this day he passed $6 \frac{1}{2} \mathrm{oz}$. more, showing a smaller amount of albumen (one fourth) and a larger proportion of urea ( 1 per cent.) ; a few casts were seen. The uræmic symptoms naturally diminished, but the general condition became worse. Hiccough set in, and the vomiting, which had been very troublesome for some days, preventing all attempts at feeding by the mouth, now became incessant. He lived till the 26th (five days), passing each day about $30 \mathrm{oz}$. of urine, which became nearly clear, containing but little pus and a few casts. The percentage of urea steadily increased, reaching 1.85 per cent. on the 25 th, and the albumen remained small in amount (usually about one tenth) but he did not rally at all and died exhausted by the constant hiccough and vomiting.

At the post-mortem we found the right kidney dilated and nodular, of a large size and quite soft, so that it was easy to understand how it happened that it was impossible to feel it during life, and to detect it when the perinephritic abscess was opened, for even at the post-mortem examination it felt like a piece of intestine. Some kidney substance remained, and the pelvis, which was converted into a large branched cavity contained a large quantity of pus, and the mucous membrane was thickened from old inflammation. The ureter was much distended throughout and half way down it was obstructed by a stone 1.25 inches long and $\cdot 5$ inch in diameter, which had evidently occupied this position for a long time; but it is remarkable that the distension, though not so great, is very palpable below the obstruction. This is not now very apparent owing to the effect of the spirit upon the preparation.

The left kidney was large and congested; but to the naked eye it showed but little sign of disease, and the 
pelvis was quite free from inflammation, showing clearly that there had been no obstruction on this side. The left ureter was normal and patent. The only abnormalities visible to the naked eye consisted in a few small cysts exposed by removing the capsule, and in the fact that the cortical part was perhaps proportionally rather small and the whole kidney substance somewhat opaque.

The bladder appeared to be quite healthy. The urethra was not examined, but as the urine up to the last passed without difficulty, it may be assumed that there was no obstruction in it.

There was no sign of tubercle in any organ examined.

It was interesting to note that the adhesions, resulting from the left pleurisy which occurred thirteen years previously, were quite soft and spongy, and could be easily broken down; and also that the pericardium was throughout adherent by adhesions of similar density. No suspicion of this condition was raised during life, though the heart was frequently examined. There was, indeed, as has been mentioned, a slight mitral murmur, though the mitral valve seemed to be perfectly healthy and competent. The heart was not hypertrophied, the left ventricle was perhaps a little thicker than normal, but the right was certainly thinner. This condition no doubt originated at the same time as the pleurisy, namely, thirteen years before.

Under the microscope the left kidney shows signs of chronic interstitial inflammation, in increase of the connective tissue between the tubules and around the Malpighian capsules; but there is little if any evidence of acute interstitial change. The epithelium, on the other hand, is swollen and cloudy, in some places quite plugging the tubules, in others being partly shed; these changes are no doubt to some extent chronic but principally acute. The kidney must therefore be considered to be in the state which we recognise as one that is particularly apt after operations about the bladder and urethra to develop into a surgical (suppurating) kidney ; but it is, I think, clear that it was a good working kidney which might have continued func- 
tional for a very considerable time. The stone consisted of uric acid coated by a thin layer of phosphates.

I scarcely venture to refer to the dilatation of the right ureter because its appearance has been so much changed by the spirit in which it has been preserved. Nothing will, I think, be found in the specimen to account for this dilatation below the point of obstruction, and yet this was, at the time of making the post-mortem, very marked. It cannot be supposed that the presence of the stone had set this up, for this is opposed to the experience of other cases in which it is found small below the seat of obstruction. I would suggest that it depended apon previous obstruction at the entrance of the ureter into the bladder, either by inflammatory thickening of the mucous membrane, or by the presence of plugs of pus or mucus ; in fact that the dilatation occurred before the stone began its descent. It will, I think, be allowed that dilatation of the ureter may occur without obvious mechanical causes. Such instances have been met with post mortem, and as possible examples of the same condition observed during life I might mention two cases of pyonephrosis with dilated ureters, on which I have operated by incising the kidney, in which no cause of obstruction is known. In one, a large instrument could be passed for a long distance down an enormously dilated ureter and no distension can be found on vaginal examination. The discharge from the wound in this case has, after three years, ceased, and it is clear that all the secretion from this kidney now finds its exit by the natural channel, or else that the diseased kidney has ceased to secrete urine at all, which is highly improbable.

But the point of all others which makes this case noteworthy is the remarkable sympathy exhibited on two occasions between the two kidneys; for it is clear that both in the first and second attacks the sound kidney suffered severely. In the first, the obstruction to the right ureter had clearly been overcome, at least in part, and the remaining lesion consisted practically only in a collection 
of pus around the damaged organ; but the concomitant symptom, and apparently the result, was a secretion of highly albuminous urine containing very large numbers of hyaline and granular casts. As soon, however, as the pus was evacuated, the albumen began to diminish coincidently with the fall of temperature, so that in a very few days it was no more than the pus would account for, and the casts had entirely disappeared.

On the second occasion there were two factors at work, one the obstruction of the right ureter, and the other the presence of a certain amount of morphia in the system, which, when the left kidney struck work, could only be eliminated by the perspiration and the breath. The result was absolute suppression for nearly a week, not accompanied by the symptoms of uræmic intoxication which are met with in acute Bright's disease, but by those which we are accustomed to associate with some mechanical obstruction to both ureters, $i$. e., to borrow the term used by Sir William Roberts, there were symptoms of obstructive rather than of non-obstructive suppression. We thought we were justified in assuming that such a cause existed, and it is of the greatest importance to know that this was not the case ; for it is at least probable that if the distension of the right kidney had been relieved by an early incision, the function of the left would have been restored. It was the assumption that the obstruction was mechanical which led us to believe that the damaged kidney was the only one which was functional, and that prevented me from undertaking what would certainly have been a dangerous, and probably, as was then thought, a useless operation. How far the morphia was an active cause of the suppression cannot be accurately estimated, but it is noticeable that, two days after the suppression set in, the patient had remarkably the appearance of a man suffering from opium poisoning, and that the drowsiness and contraction of the pupils certainly appeared earlier than is usual in suppression due to a mechanical cause. 
It might, of course, be maintained that, both on the first and the second occasion, acute inflammatory processes may have been at work in the left kidney, and have thus influenced the secretion. In answer to which it can only be stated that on neither occasion was the urine smoky, and that after death the only appearance of acute change was shown in the cloudy swelling of the epithelium, which, after such an illness, one would naturally expect to find.

It was suggested that the plan so ably urged and practised by Mr. Thornton should be adopted in this case, viz. that the kidneys should be explored through an abdominal incision. Had this been done, the left kidney would certainly have been felt to be enlarged, but I do not think it would have been possible to make out the right one because it was as soft as a piece of intestine. If the stone had been detected it might possibly have been pushed up into the kidney and removed through the loin with the best results, but if, as is not improbable, it had not been discovered, we should have been left more perplexed than before.

Lastly, it may be asked why, when the secretion became re-established, the patient did not recover? This is a question not easily answered. The percentage of urea was never more than 1.85 , and the quantity of urine in the twenty-four hours never more than $32 \mathrm{oz}$. It is thus clear that the kidney was not doing its proper amount of work, presumably not enough to put the patient in a condition to overcome the tendency to vomiting and hiccough which finally exhausted him.

To recapitulate, then, the points which seem to make this isolated case worthy of record, it appears probable from a consideration of the symptoms observed:

1st. That the presence of an abscess round a damaged kidney may cause intense albuminuria, and the occurrence of a large number of casts in the secretion of the opposite, comparatively healthy organ, which symptoms rapidly disappear on the evacuation of the pus. 
2nd. That irritation in such a diseased kidney (aided, perhaps, by the presence of some morphia in the system) may cause absolute suppression of urine lasting for a week, similar to that caused by mechanical obstruction, and not resembling that which occurs in the course of Bright's disease.

And it is interesting to note:

3rd. That in this instance the whole of the ureter was dilated, although the stone was lodged half-way down it.

\section{Appendix.}

The distinction between non-obstructive and obstructive suppression of urine was first clearly pointed out by Sir William Roberts, ${ }^{1}$ and is lucidly explained by the late Dr. Hilton Fagge. ${ }^{2}$ Non-obstructive suppression, which occurs in acute Bright's disease, the later stages of chronic Bright's disease, in certain acute fevers, in the algide stage of cholera, and after instrumentation of the urethra, when the kidneys are diseased and perhaps in other conditions, is usually fatal if unrelieved in two or three days, and though it is impossible to give a clear account of the symptoms caused by it which shall fit all cases, it may be said to be characterised by headache, vomiting, the rapid onset of unconsciousness, and what are known as uræmic symptoms, while any urine that may be passed is either bloody or highly albuminous, and there is often more or less anasarca.

In obstructive suppression which follows the complete blocking of both ureters, " the patient, instead of dying within a day or two, goes on for seven or eight days without any other grave symptoms, so that both he himself and his relations find it easy to imagine that there

1 'Roberts on the Urine,' 3rd edition, pp. 24 and 26.

2 Fagge's ' Principles and Practice of Medicine,' 1886, vol. ii, p. 387.

- Dr. Fagge, loc. cit. 
cannot be anything serious the matter with him. He is calm and free from distress, with an unclouded intellect, and with natural pulse, respiration, and temperature. He may be able to take food fairly well, the tongue being clean, and there being neither nausea nor vomiting. The muscular strength, however, may be observed to fail, and there is often marked sleeplessness at night. There is no desire to micturate, and sometimes no urine at all is voided. Generally, however, at very irregular intervals, the bladder discharges a fow ounces, or even a pint or more of urine. This is always pale and watery, and of very low specific gravity, and unless blood be mixed with it, is usually quite free from albumen. At the end of about a week symptoms appear which are commonly followed by a fatal termination within two or three days at the latest. The most distinctive of these are muscular twitchings, which Dr. Roberts says are never wanting. Contraction of the pupils also constantly occurs. The muscular weakness now rapidly increases, and, as a result of its involving the respiratory muscles, the breathing is slow, panting, and laborious. The appetite is entirely lost, and the tongue and the palate become dry. There is increasing drowsiness, with short fitful snatches of sleep and a little rambling delirium. Convulsions and coma rarely set in, the intellect being commonly preserved to the last, so that the patient has in more than one instance spoken sensibly the instant before his death. Diarrhœa is of quite exceptional occurrence, and so is severe vomiting. The skin is moist, and often sweats profusely. There is never any -ammoniacal or urinous odour from the surface of the skin or with the breath, nor does the body give off such odours after death. In one instance slight anasarca appeared when the suppression first took place, but it passed off entirely on the third day. The duration of life is stated by Dr. Roberts to be as a rule from nine to eleven days, and he remarks that the passing of a few ounces or even of two or three pints of a dilute urine does not seem to prolong it by more 
than a few hours. He knows of only three instances in which the patient survived beyond the eleventh day." .

I have thought it best to insert this quotation because we thus obtain a résumé of the opinion of two most careful observers, Sir William Roberts and the late Dr. Hilton Fagge. If I ventured to differ from this description in any way it would be by suggesting that death may take place as the result of a single convulsive attack, and also that the profuse perspiration described is not a constant symptom. In the body of the paper want of space has prevented me from describing very fully the patient's appearance, but almost every word of the above quotation would apply to it most accurately, especially that which describes the symptoms of the later stages of obstructive suppression.

(For report of the discussion on this paper, see 'Proceedings of the Royal Medical and Chirurgical Society,' New Series, vol. ii, p. 229.) 